\title{
Pregnancy after atrial repair for transposition of the great arteries
}

\author{
M Genoni, R Jenni, S P Hoerstrup, P Vogt, M Turina
}

\begin{abstract}
Objective-To investigate the risk of pregnancy in patients with transposition of the great arteries (TGA) who have undergone atrial repair.

Design-Retrospective analysis (1962-94) of 342 TGA patients who underwent atrial repair. Of 231 known late survivors, 48 were women over 18 years old who were interviewed about possible reproductive plans and previous pregnancies. As a control, comparison was made with data of 57500 women (mean age 26 years) obtained from the Swiss Statistical Bank in Bern.
\end{abstract}

Results-Mean follow up was 13.7 years; $66 \%$ remained asymptomatic, $29 \%$ had mild to moderate cardiac symptoms, and $5 \%$ suffered from severe cardiac symptoms (New York Heart Association grade III-IV). Thirty six of the 48 women wished to bear children and, to date, there have been 10 live births, two spontaneous first trimester abortions, and one induced abortion at 16 weeks. During pregnancy there was one case of cardiac deterioration and two cases of pneumonia. There was no evidence of congenital heart disease in the children.

Conclusions-In this relatively small series the completion of pregnancy in women with TGA who had undergone atrial repair and who had normal functional cardiac status was uncomplicated (Heart 1999;81:276-277)

Keywords: congenital heart disease; pregnancy; transposition of the great arteries; atrial repair

Survival into adulthood of women with congenital cardiovascular malformations is often a result of surgical intervention. Such reparative surgery therefore not only increases lifespan, but also allows an increasing number of women with initially fatal disorders to reach reproductive age. For patients with transposition of the great arteries (TGA), atrial repair (described by Senning in $1958^{1}$ ) has led to a major improvement in survival. The risk of pregnancy in this patient population, however, requires further evaluation.

\section{Methods}

Between 1962 and 1994, 342 patients with TGA underwent atrial repair at University Hospital Zurich (anatomical repair was the treatment of choice for TGA from 1992). Of the 231 known late survivors, 48 were women more than 18 years old, mean age 26 years. (We

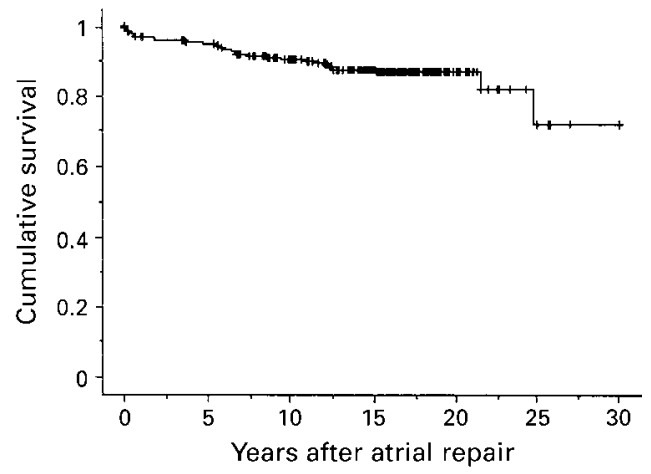

Figure 1 Actuarial survival following atrial repair for transposition of the great arteries $(n=231$ surviving patients); Kaplan-Meier analysis.

presumed that underage patients in our cohort would not report pregnancy.) These women were interviewed regarding their wish to become pregnant and about any previous pregnancies. All case records of the initial hospital admissions were reviewed.

As a control, these data were compared with information on marital status and birth from a similar population of women, namely the 57500 women aged 26 years who were living in Switzerland in 1993, which was obtained from the Swiss Statistical Bank in Bern. ${ }^{2}$

\section{Results}

The actuarial survival for the patients surviving at least 30 days after atrial repair for TGA is shown in fig 1: 90\% survived more than 10 years and $87 \%$ more than 20 years. The number of patients included in the analysis means that the data are statistically representative for 20 years; 178 patients were followed up for 10 years, 116 for 15 years, and 26 for 20 years. The mean New York Heart Association (NYHA) functional classification at a mean follow up time of 13.7 years was $1.32,66 \%$ were asymptomatic, $29 \%$ had mild or moderate cardiac symptoms, and 5\% had severe and handicapping cardiac symptoms, classifying them as NYHA III-IV.

Forty eight late survivors were women more than 18 years old, all of whom were of reproductive age, and 36 of whom wished to bear children. To date, 11 women have reported a pregnancy. There have been 10 live births, two spontaneous first trimester abortions, and one abortion at 16 weeks which was recommended by a gynaecologist in a totally asymptomatic patient. Spontaneous labour at 36 to 41 weeks occurred in seven of the live births and three were induced at 40 to 42 weeks. Vaginal delivery occurred in eight infants, while two were delivered by caesarean
Accepted for publication 13 July 1998 


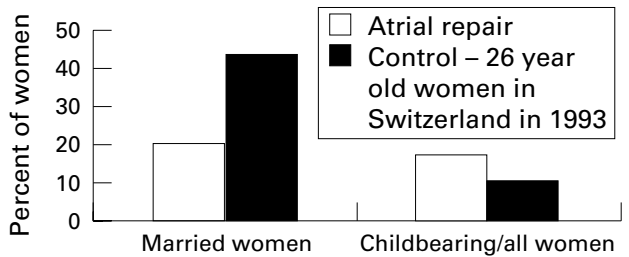

Figure 2 Marital status and births: comparison of women who had undergone atrial repair with a control group consisting of 26 year old women living in Switzerland in 1993.

section for fetal indications. There were six male and four female infants and all body weights were appropriate for the gestational age of the infants.

All except one patient were in NYHA functional classification I or II before pregnancy. During pregnancy, there was one case of deterioration in exercise tolerance and two cases of dyspnoea. The woman who experienced deterioration in exercise tolerance developed a pulmonary haemorrhage in the seventh month of pregnancy, most probably because of systemic heart failure with a decrease in the systolic area shortening fraction below $25 \%$. After undergoing a caesarean section, she initially recovered very well; however, at 12 months postpartum there was increasing deterioration of the systemic ventricle and she finally died from heart failure 19 months after giving birth. Both patients with dyspnoea recovered fully.

No arrhythmias were noted by the patients during the pregnancies.

The 10 children were born to eight $(17 \%)$ of the 48 late female survivors included in the study. Comparison with the control group (fig 2) showed that women who had undergone atrial repair had a lower rate of marriage $(20 \%$ $v 43 \%)$ but a comparable rate of birth $(17 \%$ v $10 \%)$.

There was no clinical evidence of congenital heart disease in the offspring or at follow up (12 weeks to six years).

Echocardiographic studies were performed in five of the eight patients before and after pregnancy. Only in the patient with deterioration in exercise tolerance was a decrease in right ventricular function observed, with a decrease in the systolic area shortening fraction below $25 \%$ and an increase of the end diastolic area of the systemic ventricle during pregnancy. In all other patients the echocardiographic findings remained unchanged.

\section{Discussion}

It seems that there are no medical reasons today to advise against pregnancy in women who have undergone atrial repair and who have normal functional cardiac status. Because of the good long term results of this procedure, many patients reach reproductive age and the question of a possible pregnancy is an important factor to be considered. Thus reparative surgery not only increases the lifespan of women with potentially fatal congenital malformations, but also provides the chance for a considerable number of them to give birth. The good functional results seen in our patient group means that the majority are able to pursue a "normal" life. In this context, the finding that $75 \%$ wish to have children emphasises the importance of this issue.

Although a woman's cardiac reserve may be compromised by the haemodynamic burden of pregnancy, this situation can almost always be successfully countered by addressing factors which aggravate maternal heart disease and reduce circulatory reserve, including anxiety, sodium and water retention, sudden strenuous or isometric exercise, heat and humidity, anaemia, infections, cardiac rhythm disturbances, and thromboembolic predisposition. ${ }^{3}$

One particular problem after atrial repair is the potential failure of the systemic (right) ventricle, ${ }^{45}$ which can be induced by volume overloading during pregnancy. However, in our study, only three patients had possible signs of functional heart deterioration. Moreover, with respect to these signs, Clarkson et al showed an increase in right ventricular end systolic and diastolic volume from postpartum day 1 , which returned to normal 8 to 11 weeks after delivery. ${ }^{6}$

The good outcome of pregnancy in our patients has enabled us to encourage women who want to have children to become pregnant. Nevertheless, this encouragement should be limited to patients with normal exercise tolerance and good functional status. The only case of deterioration in exertion tolerance during pregnancy in our study occurred in a patient of NYHA class II-III status before pregnancy. This patient showed good recovery after four months but later developed increasing deterioration of the systemic ventricle and died 19 months postpartum. All other patients were NYHA class I-II before pregnancy.

The risk of congenital heart disease in the children of mothers with congenital disease is higher than in the general population. This risk varies between $2 \%$ and $18 \%$ for different types of congenital heart disease, although the risk in patients with TGA remains unknown. ${ }^{7}$ However, there was no evidence of an increased risk in our patient population (10 live infants) or in other publications. Thus all the available data suggest ${ }^{6}$ that the risk of congenital heart disease in the children of mothers with TGA is at the lower end of this range. However, further studies and analyses are necessary in order to provide more detailed information about this specific patient population, so that women can be given an accurate prognosis.

1 Senning A. Surgical correction of transposition of great vessels. Surgery 1959;45:966-80.

Schweizerisches Bundesamt für Statistik. Bern, 1993: $47-59$.

3 Perloff J. Pregnancy and congenital heart disease. $\mathcal{F}$ Am Coll Cardiol 1991;18:339-42.

4 Siebenmann R, von Segesser L, Schneider K, et al. Late failure of systemic ventricle after atrial correction for transposition of great arteries. Eur f Cardiothorac Surg 1989;3:11924.

5 Turina M, Siebenmann R, Nussbaumer P, et al. Long-term outlook after atrial correction of transposition of great arteries. $\mathcal{F}$ Thorac Cardiovasc Surg 1988;95:828-35.

arteries. F Thorac Cardiovasc Surg 1988;95:828-35.
6 Clarkson P, Wilson N, Neutze J, et al. Outcome of pregnancy after the Mustard operation for transposition of the great arteries with intact ventricular septum. $7 \mathrm{Am}$ Coll Cardiol 1994;24:190-3.

7 Pitkin R, Perloff J, Koos B, et al. Pregnancy and congenital disease. Ann Intern Med 1990;112:445-54. 